\title{
The Effectiveness of Budesonide Nasal Irrigation After Endoscopic Sinus Surgery in Chronic Rhinosinusitis With Asthma
}

\author{
Tae Wook Kang $\cdot$ Jae Ho Chung $\cdot$ Seok Hyun Cho $\cdot$ Seung Hwan Lee $\cdot$ Kyung Rae Kim $\cdot$ Jin Hyeok Jeong \\ Department of Otolaryngology-Head and Neck Surgery, Hanyang University School of Medicine, Seoul, Korea
}

Objectives. Budesonide nasal irrigation was introduced recently for postoperative management of patients with chronic rhinosinusitis. The safety and therapeutic effectiveness of this procedure is becoming accepted by many physicians. The objective of this study was to evaluate the efficacy of postoperative steroid irrigation in patients with chronic rhinosinusitis and asthma.

Methods. This prospective study involved 12 chronic rhinosinusitis patients with nasal polyps and asthma who received oral steroid treatment for recurring or worsening disease. The 22-item Sinonasal Outcomes Test (SNOT-22) and LundKennedy endoscopy scores were checked before nasal budesonide irrigation, and 1, 2, 4, and 6 months after irrigation. We also calculated the total amount of oral steroids and inhaled steroids in the 6 months before irrigation and the 6 months after it.

Results. The mean SNOT-22 score improved from $30.8 \pm 14.4$ before irrigation to $14.2 \pm 8.7$ after 6 months of irrigation $(P=0.030)$. The endoscopy score also improved from $7.4 \pm 4.7$ before irrigation to $2.2 \pm 2.7$ after 6 months $(P<0.001)$. The total amount of oral steroid was decreased from $397.8 \pm 97.6 \mathrm{mg}$ over the 6 months before irrigation to $72.7 \pm$ $99.7 \mathrm{mg}$ over the 6 months after irrigation $(P<0.001)$.

Conclusion. Nasal irrigation with budesonide is an effective postoperative treatment for chronic rhinosinusitis with asthma, which recurs frequently, reducing the oral steroid intake.

Keywords. Nasal Lavage; Budesonide; Sinusitis; Asthma; Nasal Polyp

\section{INTRODUCTION}

Chronic rhinosinusitis (CRS) with nasal polyp is a common inflammatory condition of the nasal mucosa carrying significant morbidity and detriment to quality of life (QOL). Many CRS patients with nasal polyps require surgery. Along with the development of new equipment and technology, the resolution of the endoscope has increased resulting in a decrease in recurrence of

\footnotetext{
- Received February 1, 2016

Revised May 3, 2016

Accepted May 30, 2016

- Corresponding author: Jin Hyeok Jeong

Department of Otolaryngology-Head and Neck Surgery, Hanyang

University School of Medicine, 222 Wangsimni-ro, Seongdong-gu, Seoul 04763, Korea

Tel: +82-31-560-2368, Fax: +82-31-560-2894

E-mail: ent@hanyang.ac.kr
}

the disease. Unfortunately, even after surgery the patients with asthma are often difficult to manage because of the high recurrence rate and worse clinical course. We have to explain to patients with asthma before the surgery that endoscopic sinus surgery (ESS) is not intended to be curative but is rather directed towards long-term symptom control [1].The current mainstay of medical therapy for CRS with asthma after surgery is saline irrigation, topical or systemic steroid or recurrent oral medication of some other kind. In severe cases of recurrence, revision surgery may be required.

Topical nasal steroid spray can be effective in patients with CRS: the nasal polyps may decrease in size and not recur [2]. But the effect is not constant especially in those with asthma, so systemic steroid is often used in such cases [1].The side effect of longterm systemic steroid are of serious concern to physicians [3].

The low efficiency of topical steroid sprays might be because

Copyright $@ 2017$ by Korean Society of Otorhinolaryngology-Head and Neck Surgery.

This is an open-access article distributed under the terms of the Creative Commons Attribution Non-Commercial License (http://creativecommons.org/licenses/by-nc/4.0)

which permits unrestricted non-commercial use, distribution, and reproduction in any medium, provided the original work is properly cited. 
little of the steroid is actually delivered to the severely inflamed sinus mucosa and polyps. Other reasons could be swelling of the mucosa, recurrence of polyps in asthma patients or the production of a jelly-like thick discharge, often observed in asthma patients, all of which could prevent the drug reaching the deep sinus mucosa. Recently, delivery of steroid in a large volume by nasal irrigation with high pressure mechanism has been recommended as a better option for treating chronic sinus mucosal inflammation than using a low volume with low pressure [4]. A post-sinus surgery corridor is essential especially for long-term treatment of recalcitrant CRS, to permit exposure of the drug to the mucosa, since the nasal irrigation solution can be successfully delivered via such a corridor [2].

The off-label use of budesonide nasal irrigation was introduced recently for postoperative management of patients with CRS. The safety and effectiveness of this procedure is becoming accepted by many physicians $[2,5-8]$. The objective of this study was to figure out if budesonide nasal irrigation could reduce the repeated use of oral steroid in recalcitrant CRS patients with asthma.

\section{MATERIALS AND METHODS}

\section{Subjects}

The subjects of this study were 12 asthma patients who underwent ESS from 2012 to 2014 and took repeated topical or systemic steroid over 6 months due to recurrence or worsening of the disease after ESS. Two patients (17\%) had their surgery in another hospital. Out of the 12 asthma patients, 7 patients were diagnosed as asthma and treated in Hanyang University Guri Hospital while other 5 patients were diagnosed and treated in other hospitals. History of allergic rhinitis in the subjects were investigated as well. Thirty milligrams of oral steroid were prescribed daily for the patients with aggressive nasal symptom and they were tapered during a week. Fifteen milligrams of oral steroid were prescribed daily for 1 week without tapering when the patients complained moderate nasal symptom. All the patients had used saline nasal irrigation continuously. The prospective study was performed, and the medication histories were obtained, by chart review. The study was approved by the Institutional Review Board of Hanyang University Guri Hospital and

\section{H I I G H L I I G H T T S}

- Budesonide nasal irrigation improved the mean SNOT-22 and endoscopic score.

- The total amount of oral steroid for sinusitis control decreased.

- The use of inhaled steroid for asthma control did not decrease.

- Budesonide nasal irrigation is an effective postoperative treatment for sinusitis in asthma patients. informed consent was obtained from all participants prior to the study (IRB No. 2014-09-001-002).

\section{Budesonide nasal irrigations}

Squeeze bottles ( $250 \mathrm{~mL}$; Nose sweeper bottle, Medicore Inc., Uijeongbu, Korea) were used. Irrigation with normal saline (Nose sweeper powder, Medicore Inc.) was carried out prior to the budesonide irrigation. Then, one pack of $0.5 \mathrm{mg} / 2 \mathrm{~mL}$ commercially available generic budesonide respules (Pulmican Respules, Kuhnil Inc., Seoul, Korea) was mixed into a squeeze bottle with $250 \mathrm{~mL}$ normal saline. Half of the solution was used for each nasal cavity. At the end the patient gargled with regular water to prevent irritation of the oral cavity mucosa and candida infection. Irrigation was done twice a day for 6 months.

During budesonide nasal irrigation, we didn't use any other medication for CRS treatment except systemic steroid using periods.

Inclusion criteria were the following; age over 18 years, receiving treatment for asthma after being diagnosed, and having received over 6 months of medical management after surgery due to repetitive worsening or recurrence of CRS with nasal polyps. Exclusion criteria were: corticosteroid use for conditions other than CRS with nasal polyps, and suffering from diseases that were relative or absolute contraindications for steroid use.

\section{Outcome measures}

Both subjective and objective outcome measures were used.The primary outcome measure was a change in QOL based on the 22-item Sinonasal Outcomes Test (SNOT-22). The secondary outcome was a change in Lund-Kennedy endoscopy (LK) score. To minimize bias, a single physician measured the scores. A baseline study was carried out before the nasal budesonide irrigation, and this was followed-up 1, 2, 4, and 6 months after the irrigation in the outpatient clinic.

We also calculated the total amount of oral steroids and inhaled steroids used by the patients and compared the amounts used in the 6 months before and after irrigation. Total amounts of oral steroid taken for sinus control were measured in milligrams, and total amounts of steroids inhaled for asthma control were recorded as numbers of bottles in each case using as source the prescription orders for the patients in our hospital.

\section{Statistics}

We used IBM SPSS ver. 21.0 (IBM Co., Armonk, NY, USA).Values are means \pm standard deviation unless otherwise indicated. In the SNOT-22 and LK study, the Kruskal-Wallis method was used to evaluate differences, and post hoc comparisons were made with the Mann-Whitney $U$-test. Oral and inhaled steroid use before and after irrigation was compared with the MannWhitney $U$-test. Differences were considered significance at $P<0.05$. 


\section{RESULTS}

The mean period of budesonide nasal irrigation was $4.8 \pm 0.9$ months. This is because, even though we offered 6 months of irrigation to all patients, some of them skipped sessions and tended to leave gaps from time to time for personal reasons.

\section{Patient population}

The subjects were 3 males and 9 females. Mean age was 49.9土

Table 1. Characteristics of the patient population $(n=12)$

\begin{tabular}{lc}
\hline Characteristic & No. $(\%)$ \\
\hline Age (yr), mean \pm SD & $49.9 \pm 0.9$ \\
Female gender & $9(75)$ \\
Nonsmoker & $9(75)$ \\
Revision surgery & $1(8)$ \\
\hline
\end{tabular}

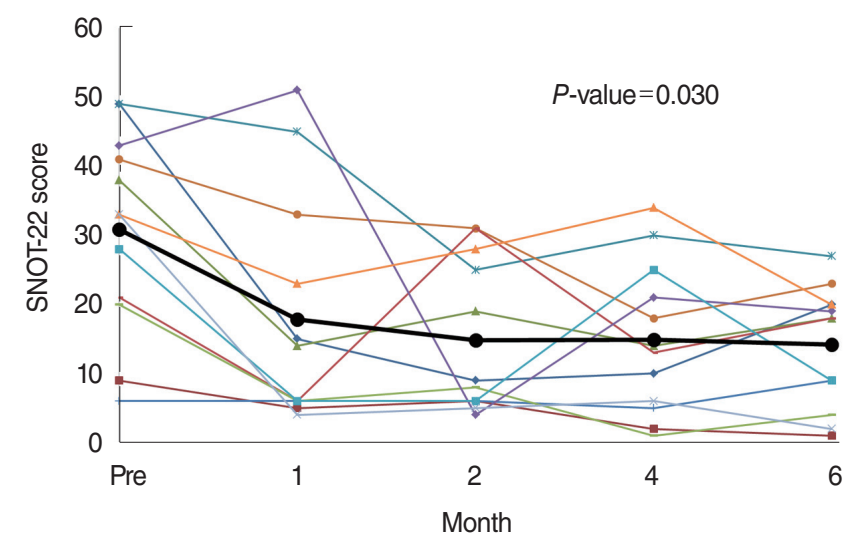

Fig. 1. Comparison of 22-item Sinonasal Outcomes Test (SNOT-22) scores before and after budesonide nasal irrigation. The mean SNOT-22 score (black circles and bold line) was significantly lower at each follow-up than before the irrigation $(P$-value $=0.030)$. Black bold circle mean of patients.

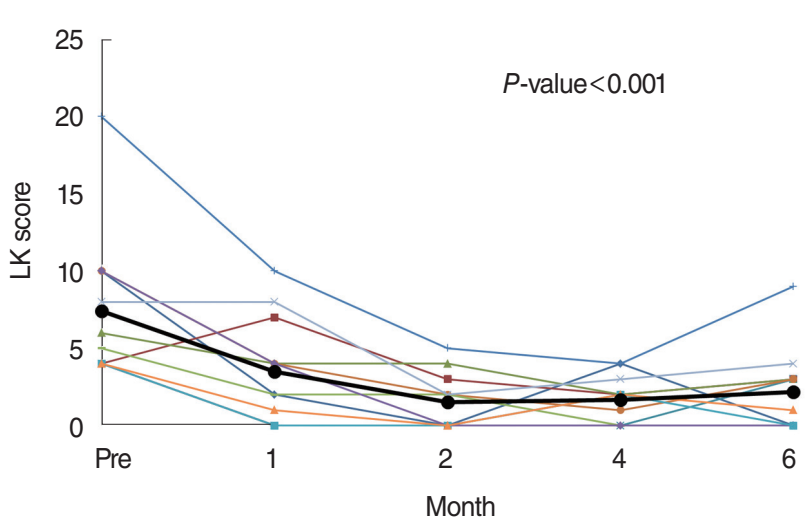

Fig. 2. Comparison of Lund-Kennedy endoscopy (LK) scores before and after budesonide nasal irrigation. The mean LK score (black circles and bold line) was significantly lower at each follow-up than before the irrigation $(P$-value $<0.001)$. Black bold circle mean of patients.
7.3 years. Eleven of the patients (92\%) had histories of allergic rhinitis (Table 1).

\section{SNOT-22 score}

The mean baseline SNOT-22 score was $30.8 \pm 14.4$ before budesonide irrigation, and the score was $17.8 \pm 15.9$ after 1 month, $14.8 \pm 10.6$ after 2 months, $14.9 \pm 10.4$ after 4 months and $14.2 \pm 8.7$ after 6 months. There was a statistically significant difference between before and after budesonide irrigation $(P=0.030)$ (Fig. 1).

\section{Lund-Kennedy endoscopy scores}

The baseline LK score was $7.4 \pm 4.7$ before budesonide irrigation and the average score was $3.5 \pm 3.2$ after 1 month, $1.5 \pm 1.7$ after 2 months, $1.7 \pm 1.4$ after 4 months and $2.2 \pm 2.7$ after 6 months of budesonide irrigation. There was a statistically significant difference between before and after budesonide irrigation $(P<0.001)$ (Fig. 2).

\section{Total amount of oral steroids}

The total amount of systemic steroid used in the 6 months before budesonide irrigation was $397.8 \pm 97.6 \mathrm{mg}$, and for the 6 months after budesonide irrigation it was $72.7 \pm 99.7 \mathrm{mg}(P<$
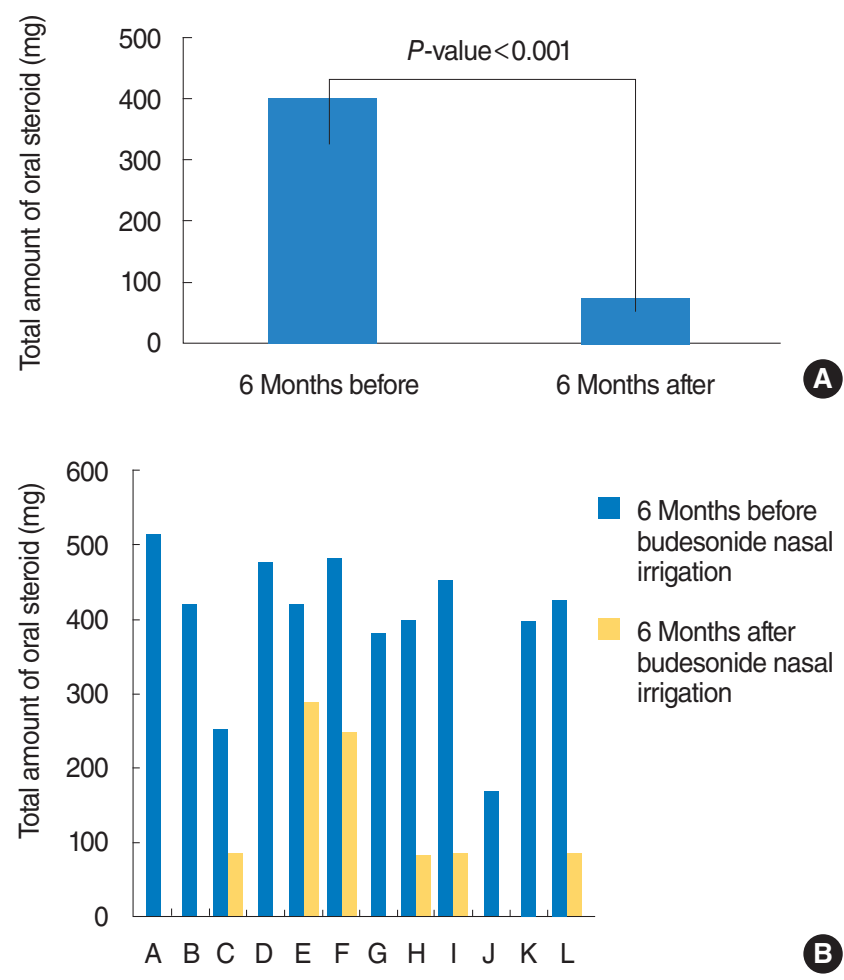

Fig. 3. Comparison of total amounts of oral steroid before and after budesonide nasal irrigation. (A) Average amounts of total oral steroid $(P$-value $<0.001)$. (B) Total amounts of oral steroid used by each patient. Six patients took no systemic steroid after budesonide irrigation, and the other 6 patients used less steroid after the irrigation. 


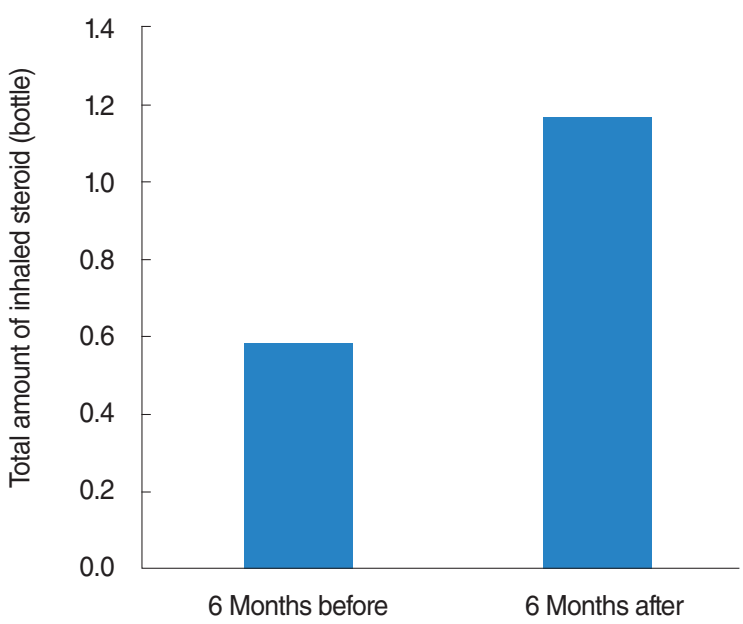

Fig. 4. Comparison of total amounts of steroid inhaled for asthma before budesonide nasal irrigation and after. They showed no significant difference between each other $(P=0.198)$.

0.001) (Fig. 3). Six of the 12 patients (50\%) took no systemic steroid after the irrigation, while the other 6 used significantly less steroid after the irrigation.

\section{Amount of inhaled for asthma}

The use of inhaled steroid was an average of $0.6 \pm 0.9$ bottles for the 6 months before irrigation and $1.2 \pm 1.2$ bottles for the 6 months after irrigation, but the difference was not statistically significant $(P=0.198)$ (Fig. 4).

\section{DISCUSSION}

According to this study, budesonide nasal irrigation in asthma patients was able to reduce the repeated use of oral steroid but not the use of inhaled steroid. Most cases of CRS with nasal polyps recover after ESS, but, when accompanied by asthma the disease tends to worsen repetitively or recur often. In such cases, constant management along with treatment of the asthma is essential $[9,10]$. In those with asthma, repeated treatments such as nasal saline irrigation, nasal steroid spray, oral steroid or antibiotics, and even operation, are not rare. These procedures, when repeated, can result in reduced QOL and put patients in serious danger of side effects due to the use of numerous drugs. Our findings show that constant nasal irrigation with budesonide solution twice a day can prevent worsening of the disease and so improve QOL, and even reduce systemic steroid consumption.

A recent meta-analysis showed that topical steroid is effective against sinonasal symptoms in patients with CRS without nasal polyps [11], and it decreases polyp size [12,13] and prevents polyp recurrence in CRS with nasal polyps [12]. The literature contains a number of different findings concerning the use of nasal steroid spray after ESS. Dijkstra et al. [14] used a double- blinded randomized trial to assess the use of fluticasone propionate in reducing polyposis recurrence after ESS. The conclusion of the study was that the steroid did not have any beneficial effect on disease recurrence. Lavigne et al. [15] examined a similar situation in revision ESS patients, using delivery of the steroid through a maxillary antrostomy indwelling tube, and concluded that budesonide was superior to placebo. Virolainen and Puhakka [16] reported that beclomethasone was beneficial in decreasing polyp recurrence postoperatively and in improving patient symptoms. Most of the above studies did not distinguish between patients with and without asthma.

Delivery technique, the surgical state of the sinus cavity, the delivery device and fluid dynamics have significant impacts on the delivery of topical therapies to the sinus mucosa [17]. It is likely that the reason for poor absorption of the nasal steroid spray is that the medication does not reach the diseased sinus mucosa [18]. Nasal steroid sprays are effective in conditions such as allergic rhinitis and when polyps protrude into the nasal airway [2]. For a long-term CRS treatment, a post-sinus surgery corridor is essential to provide drug exposure to sinus mucosa $[19,20]$. After ESS this becomes even more difficult because the open area that needs to be targeted is both larger and deeper in the paranasal sinus cavity [1]. Thus, saline nasal irrigation is known to be more effective than a saline spray in improving the symptoms of CRS. Pynnonen et al. [21] showed in a randomized controlled trial that patients who received saline irrigation in a large volume and at low positive pressure received significantly greater benefit than those who received a nasal saline spray in terms of QOL. Wormald et al. [22] used a Technetium$99 \mathrm{~m}$ sulfur colloid tracer to compare a metered nasal spray, nebulization with RinoFlow and nasal douching in patients with CRS who underwent ESS. The accumulation of radioactivity was assessed and nasal irrigation was found to be the most effective in penetrating the maxillary sinus and frontal recess. The greater efficacy of irrigation over saline spray may be due to the larger volume and increased delivery pressure which may allow topical steroids to more effectively contact the sinus mucosa and enhance the mechanical removal of mucus, inflammatory products, and bacteria/biofilms [4,17]. In a recent Cochrane review, topical steroid delivery for patients with CRS without nasal polyps led to a greater proportion of responders and more beneficial effects in symptom control when delivered directly to the sinus cavities than did simple nasal sprays [11].

The off-label use of budesonide irrigation is being rapidly accepted by physicians, and the safety and efficiency of the irrigation is also being confirmed [1,2,4-8]. Sachanandani et al. [6] applied irrigation to the nasal cavities of $0.5 \mathrm{mg} /$ respule once a day for 30 days, and the subjects showed significant clinical improvement. Welch et al. [5] applied $0.5 \mathrm{mg} / 2 \mathrm{~mL}$ of budesonide respule mixed with $240 \mathrm{~mL}$ of saline solution twice daily for 6 months, but serum cortisol and 24 hour urinary cortisol levels showed no significant decrease. Thamboo et al. [7] applied $1 \mathrm{mg}$ 
of budesonide twice daily in $120 \mathrm{~mL}$ of saline solution for 60 days, and detected no adrenal suppression in the adrenocorticotropic hormone (ACTH) stimulation test. In our study, a $0.5-\mathrm{mg}$ respule of budesonide mixed in a $250-\mathrm{mL}$ bottle of normal saline and delivered to both nasal cavities twice a day was prescribed for safety based on the above references.

Some studies support the effectiveness of budesonide nasal irrigation in CRS patients after ESS. Snidvongs et al. [2] reported on 111 patients with CRS undergoing ESS after failing previous medical therapy, and the SNOT-22 and endoscopy scores of the subjects showed significant improvement. Jang et al. [8] analyzed the effectiveness of irrigation in 60 patients comprising 30 patients with eosinophilic CRS, 13 with Samter's triad and 17 others. Postoperative budesonide nasal irrigation lowered the SNOT-20 scores significantly in all patients, but the endoscopy scores improved only in the eosinophilic CRS group. Although this has not been confirmed by biopsy, our subjects were CRS patients with asthma, mostly accompanied by eosinophilic CRS, and thus our results for budesonide nasal irrigation could be seen as applying to eosinophilic CRS.

A double-blind randomized controlled trial by Rotenberg et al. [1] compared the outcomes of postoperative treatments such as saline irrigation, budesonide nasal spray, saline irrigation mixed with budesonide nasal spray after ESS in Samter's triad patients. Unlike our results, Rotenberg et al. [1] found that budesonide nasal spray or irrigation was not superior to saline irrigation. Since our patients were diagnosed with asthma only, not Samter's triad, the results in the two cases could well be different.

The total amount of oral steroid used decreased significantly after budesonide irrigation. There has been no other study, so far, analyzing the change of systemic steroid dose after irrigation. Thus, budesonide nasal irrigation appears to be not only safe and effective, but also decreases the use of systemic steroids and reduces their side effects. Long-term use is indeed a possible danger for patients using systemic steroid [3], and the reduced systemic steroid use could well be encouraging for physicians who treat such patients.

The amount of steroid inhaled for asthma after irrigation did not differ significantly from the amount before irrigation. The subjects of our study received their treatment in other clinics before visiting our facility so the lack of a difference before and after irrigation may be because the amount of steroid inhaled before irrigation may have been underestimated. It could also mean that budesonide nasal irrigation does not influence the lower airway. Although the upper airway and lower airway are described as 'one airway or one disease' concept, our study shows that local management of the nasal cavity and sinus does not influence the accompanying asthma, which is a lower airway disease.

There are several limitations to this study. First, the number of subjects was relatively small so there was no control group. Sec- ond, the amounts of systemic and inhaled steroid taken by the patients before visiting our hospital were not under our control, and this could have influenced the results.

Unlike other studies of efficacy and safety of budesonide irrigation by subjective symptom and endoscopic finding, this study showed budesonide nasal irrigation in asthma patients was able to reduce the repeated use of oral steroid significantly but not the use of inhaled steroid.

In conclusion, among the various postoperative treatments for this recalcitrant CRS in asthma patients, budesonide nasal irrigation is an effective method for improving QOL, preventing recurrence and reducing the use of oral steroid. But there was no decrease in the use of inhaled steroid after the irrigation.

In order to encourage extensive use of budesonide nasal irrigation, further examination of the appropriate dose, frequency of irrigation, and duration of treatment is needed, along with assessment of the safety of its long-term use.

\section{CONFLICT OF INTEREST}

No potential conflict of interest relevant to this article was reported.

\section{REFERENCES}

1. Rotenberg BW, Zhang I, Arra I, Payton KB. Postoperative care for Samter's triad patients undergoing endoscopic sinus surgery: a double-blinded, randomized controlled trial. Laryngoscope. 2011 Dec;121(12):2702-5.

2. Snidvongs K, Pratt E, Chin D, Sacks R, Earls P, Harvey RJ. Corticosteroid nasal irrigations after endoscopic sinus surgery in the management of chronic rhinosinusitis. Int Forum Allergy Rhinol. 2012 Sep-Oct;2(5):415-21.

3. Poetker DM, Reh DD. A comprehensive review of the adverse effects of systemic corticosteroids. Otolaryngol Clin North Am. 2010 Aug;43(4):753-68.

4. Adappa ND, Wei CC, Palmer JN. Nasal irrigation with or without drugs: the evidence. Curr Opin Otolaryngol Head Neck Surg. 2012 Feb;20(1):53-7.

5. Welch KC, Thaler ER, Doghramji LL, Palmer JN, Chiu AG. The effects of serum and urinary cortisol levels of topical intranasal irrigations with budesonide added to saline in patients with recurrent polyposis after endoscopic sinus surgery. Am J Rhinol Allergy. 2010 Jan-Feb;24(1):26-8.

6. Sachanandani NS, Piccirillo JF, Kramper MA, Thawley SE, Vlahiotis A. The effect of nasally administered budesonide respules on adrenal cortex function in patients with chronic rhinosinusitis. Arch Otolaryngol Head Neck Surg. 2009 Mar;135(3):303-7.

7. Thamboo A, Manji J, Szeitz A, Santos RD, Hathorn I, Gan EC, et al. The safety and efficacy of short-term budesonide delivered via mucosal atomization device for chronic rhinosinusitis without nasal polyposis. Int Forum Allergy Rhinol. 2014 May;4(5):397-402.

8. Jang DW, Lachanas VA, Segel J, Kountakis SE. Budesonide nasal irrigations in the postoperative management of chronic rhinosinusitis. Int Forum Allergy Rhinol. 2013 Sep;3(9):708-11. 
9. Young J, Frenkiel S, Tewfik MA, Mouadeb DA. Long-term outcome analysis of endoscopic sinus surgery for chronic sinusitis. Am J Rhinol. 2007 Nov-Dec;21(6):743-7.

10. Kim JE, Kountakis SE. The prevalence of Samter's triad in patients undergoing functional endoscopic sinus surgery. Ear Nose Throat J. 2007 Jul;86(7):396-9.

11. Snidvongs K, Kalish L, Sacks R, Craig JC, Harvey RJ.Topical steroid for chronic rhinosinusitis without polyps. Cochrane Database Syst Rev. 2011Aug;(8):CD009274.

12. Kalish L, Snidvongs K, Sivasubramaniam R, Cope D, Harvey RJ. Topical steroids for nasal polyps. Cochrane Database Syst Rev. 2012 Dec;12:CD006549.

13. Joe SA, Thambi R, Huang J. A systematic review of the use of intranasal steroids in the treatment of chronic rhinosinusitis. Otolaryngol Head Neck Surg. 2008 Sep;139(3):340-7.

14. Dijkstra MD, Ebbens FA, Poublon RM, Fokkens WJ. Fluticasone propionate aqueous nasal spray does not influence the recurrence rate of chronic rhinosinusitis and nasal polyps 1 year after functional endoscopic sinus surgery. Clin Exp Allergy. 2004 Sep;34(9):1395-400.

15. Lavigne F, Cameron L, Renzi PM, Planet JF, Christodoulopoulos P, Lamkioued B, et al. Intrasinus administration of topical budesonide to allergic patients with chronic rhinosinusitis following surgery. La- ryngoscope. 2002 May;112(5):858-64.

16. Virolainen E, Puhakka $H$. The effect of intranasal beclomethasone dipropionate on the recurrence of nasal polyps after ethmoidectomy. Rhinology. 1980 Mar;18(1):9-18.

17. Harvey RJ, Schlosser RJ. Local drug delivery. Otolaryngol Clin North Am. 2009 Oct;42(5):829-45.

18. Bateman ND, Whymark AD, Clifton NJ, Woolford TJ. A study of intranasal distribution of azelastine hydrochloride aqueous nasal spray with different spray techniques. Clin Otolaryngol Allied Sci. 2002 Oct;27(5):327-30.

19. Snidvongs K, Chaowanapanja P, Aeumjaturapat S, Chusakul S, Praweswararat P. Does nasal irrigation enter paranasal sinuses in chronic rhinosinusitis? Am J Rhinol. 2008 Sep-Oct;22(5):483-6.

20. Harvey RJ, Debnath N, Srubiski A, Bleier B, Schlosser RJ. Fluid residuals and drug exposure in nasal irrigation. Otolaryngol Head Neck Surg. 2009 Dec;141(6):757-61.

21. Pynnonen MA, Mukerji SS, Kim HM, Adams ME, Terrell JE. Nasal saline for chronic sinonasal symptoms: a randomized controlled trial. Arch Otolaryngol Head Neck Surg. 2007 Nov;133(11):1115-20.

22. Wormald PJ, Cain T, Oates L, Hawke L, Wong I.A comparative study of three methods of nasal irrigation. Laryngoscope. 2004 Dec;114 (12):2224-7. 